\title{
Predictive value of a negative oral provocation test in patients with hypersensitivity to analgesics
}

\author{
Maja Jakičc ${ }^{1^{\star}}$, Miha Jager $^{1^{\star}}$, Mitja Košnik ${ }^{1,2 凶}$
}

\begin{abstract}
Introduction: Nonsteroidal anti-inflammatory drugs (NSAIDs) take first or second place as the cause of drug-induced hypersensitivity reactions. The oral provocation test (OPT) is a gold standard for the diagnosis of NSAID hypersensitivity. We investigated which analgesics patients took after a negative OPT and determined the proportion of patients that experienced a hypersensitivity reaction despite a negative OPT.

Methods: We selected 115 patients (67.8\% female, age $54.9 \pm 16.7$ years) with a negative aspirin OPT and a convincing history of immediate hypersensitivity to aspirin or NSAIDs. In a telephone survey, we identified the analgesics taken after the OPT and possible adverse events.

Results: The mean follow-up time was $5.1 \pm 2.0$ years. All subjects needed at least one analgesic drug. Despite the negative outcome of the aspirin OPT, only $33.9 \%$ of subjects took aspirin and $0.9 \%$ had a hypersensitivity reaction. The negative predictive value (NPV) of the aspirin OPT was $97.4 \%$. Overall, 16 (13.9\%) subjects experienced a hypersensitivity reaction, 12 of which occurred after taking a drug not tested with the OPT. The NPV of the OPT for all NSAIDs was $96.4 \%$.

Conclusion: Our results support the available data that most subjects do not re-take the tested drug regardless of the high NPV of the OPT.
\end{abstract}

Keywords: aspirin, hypersensitivity, negative predictive value, NSAIDs, oral provocation test

Received: 18 December 2015 | Returned for modification: 4 January 2016 | Accepted: 15 February 2016

\section{Introduction}

Hypersensitivity to analgesics affects approximately $3 \%$ of the general population (1-6). The vast majority of hypersensitivity reactions are non-immunologic (non-allergic) due to cyclooxygenase 1 (COX-1) inhibition by aspirin or nonsteroidal anti-inflammatory drugs (NSAIDs) $(7,8)$. Symptoms develop because of leukotriene overproduction. In addition, decreased levels of PG E2 and PG D2 enhance histamine release from mast cells $(9,10)$. This mechanism is involved in NSAID-exacerbated respiratory disease (NERD), NSAID-exacerbated chronic urticaria (NECD), and NSAID-induced urticaria-angioedema (NIUA) (8, 11, 12). Symptoms occur within 1 to 4 hours after NSAID intake. There is marked cross-reactivity among COX-1 inhibitors (7). These patients most often tolerate weak COX-1 inhibitors and COX-2 inhibitors $(3,11$, 13, 14).

In some patients, urticaria or even anaphylaxis occurs in the 1st hour after ingestion of only a single NSAID or a few NSAIDs belonging to the same chemical group (single NSAID-induced urticaria, angioedema, or anaphylaxis, SNIUAA). This type of reaction is suggestive of an immune-mediated type I reaction, although IgE and skin tests are rarely positive $(15,16)$. Nine cases of diclofenac-induced anaphylaxis were reported to the Allergy Vigilance Network in France (17).

Cell-mediated hypersensitivity reactions occur within days of taking a single NSAID (4).

Drug provocation is a gold standard in the diagnosis of NSAID hypersensitivity $(3,11)$. If the challenge with aspirin is positive, the patient is diagnosed as intolerant to COX-1 inhibitors. If the challenge is negative, the patient might have a selective NSAID allergy or be NSAID-tolerant. Provocation with the culprit drug is warranted in cases with an unclear history (4).
However, a negative oral provocation test (OPT) result does not completely exclude hypersensitivity to NSAIDs (11). False negative results might occur due to the absence of co-factors in the OPT, such as viral infection, co-medication, and physical exercise (3, 18). In addition, there is a concern that slowly increasing doses during the OPT might induce transient desensitization and thus result in a false negative test $(19,20)$.

We analyzed a group of patients that were OPT-negative for aspirin. We investigated the analgesics the patients took after the negative OPT and determined the proportion of patients that experienced a hypersensitivity reaction.

\section{Methods}

This study was approved by the national ethics committee (study no. 72/02/15). We identified patients from an OPT database that were tested for hypersensitivity to aspirin, other NSAIDs, or paracetamol between 2004 and 2014. An OPT was started with onehundredth of the therapeutic dose. Then, one-tenth, half, and a full dose were administered at intervals of 60 to 90 minutes. The test was considered positive if the peak expiratory flow decreased by at least $20 \%$ or if urticaria, angioedema, nasal stuffiness, or anaphylaxis developed.

We reviewed the medical files of patients that were subjected to an OPT for aspirin between 2007 and 2011 and in 2013. We gathered information concerning the reaction that was the reason for the diagnostic workup (index reaction), including the clinical presentation, the NSAID causing the hypersensitivity, and the possible diagnosis of asthma, nasal polyposis, and/or chronic urticaria. We obtained data for the OPT results and the NSAIDs that were identified to be safe for use after the OPT. We selected patients with a convincing personal history of immediate hyper- 
sensitivity to aspirin or NSAIDs and a negative OPT to aspirin. The criteria for a convincing history were a reaction occurring up to 4 hours after taking the drug and patients presenting with urticaria, angioedema, nasal stuffiness, dyspnea, or anaphylaxis that could not be explained by an alternative cause. We investigated whether the discharge letter clearly advised which drugs the patient should take and whether aspirin was listed among the drugs advised.

In a telephone survey, we evaluated patients' analgesic needs after the testing. We specifically asked whether they had taken aspirin and NSAIDs that had been negative on the OPT. Patients that did not take drugs that were negative on the OPT were asked the reason for not taking that drug.

\section{Statistical methods}

The data were statistically analyzed using the statistics software SPSS (Statistical Package for the Social Sciences version 22, International Business Machines Corp., Armonk, NY). The data are shown as the average and standard deviation. To compare the differences between groups, we used a $t$-test and chi-square test. The negative predictive value (NPV) of the OPT for NSAIDs and aspirin was calculated based on all patients that retook the same drug after a negative OPT.

\section{Results}

\section{The study group}

In a 6-year period, 664 subjects $(68.7 \%$ female; age $52.3 \pm 16.2$ years, range 19 to 92 ) were subjected to OPTs with analgesics. We excluded 205 subjects $(30.9 \%)$ that were not tested with aspirin and $45(6.8 \%)$ subjects with a positive outcome of the OPT. A total of 254 subjects (38.3\%) had a vague clinical history. Out of the remaining 160 subjects, we were unable to contact 36 patients and nine did not want to participate (response rate was 71.9\%).

A total of 115 subjects (67.8\% female, $54.9 \pm 16.7$ years) participated in the survey. The sex and age distribution of the patients participating in the survey were the same as the distribution in the initial unselected group, confirming that the selection of patients for the survey was not biased. Six (5.3\%) subjects had asthma, one (0.9\%) had asthma and nasal polyps, one (0.9\%) had asthma and chronic urticaria, and nine $(7.4 \%)$ had chronic urticaria.

\section{Index hypersensitivity reactions}

The characteristics of the index hypersensitivity reactions are presented in Table 1. The most common presentation was urticaria and angioedema (72.0\%). Among the patients, 51.6\% reacted to multiple COX-1 inhibitors and $13.9 \%$ convincingly reacted to a single drug (aspirin, pyrazolones, or diclofenac).

\section{Drugs taken after the OPT}

The mean follow-up time after a negative aspirin OPT was $5.1 \pm$ 2.0 years. Paracetamol was an efficient analgesic for $20.0 \%$ of subjects, and the others needed stronger analgesic therapy. Table 2 shows the details of the drugs that the subjects took after the negative OPT and whether they experienced any reaction to those drugs. Sixteen patients reported hypersensitivity reactions; however, 12 of these occurred after taking a drug that was not tested in the OPT. The most common hypersensitivity reactions were urticaria and angioedema (12 subjects, 75\%). The hypersensitivity reaction most commonly occurred after paracetamol or pyrazolones. Hypersensitivity reactions occurred in four subjects despite a negative OPT with a particular analgesic (two paracetamol, one

Table 1 | Distribution of hypersensitivity types according to non-steroidal anti-inflammatory drugs in the index reaction.

\begin{tabular}{|c|c|c|c|c|c|c|c|}
\hline \multirow[b]{2}{*}{ Drug } & \multicolumn{5}{|c|}{ Hypersensitive reaction } & \multirow[b]{2}{*}{ SUM } & \multirow{2}{*}{$\begin{array}{c}\text { No. of patients testec } \\
\text { with that drug as } \\
\text { culprit drug }\end{array}$} \\
\hline & $\begin{array}{c}\text { Asthma } \\
\text { exacerbation }\end{array}$ & Rhinitis & $\begin{array}{c}\text { Urticaria, } \\
\text { angioedema }\end{array}$ & $\begin{array}{l}\text { Anaphylactic } \\
\text { reaction }\end{array}$ & Other* & & \\
\hline Aspirin & $0(0.0 \%)$ & $2(1.7 \%)$ & $55(47.8 \%)$ & $2(1.7 \%)$ & $17(14.8 \%)$ & $76(66.1 \%)$ & $76(100 \%)$ \\
\hline Paracetamol & $0(0.0 \%)$ & $1(0.9 \%)$ & $19(16.5 \%)$ & $2(1.7 \%)$ & $5(4.3 \%)$ & 27 (23.5\%) & $22(81.5 \%)$ \\
\hline Naproxen & $0(0.0 \%)$ & $0(0.0 \%)$ & $13(11.3 \%)$ & $0(0.0 \%)$ & $1(0.9 \%)$ & $14(12.2 \%)$ & $6(42.9 \%)$ \\
\hline Diclofenac & $0(0.0 \%)$ & $1(0.9 \%)$ & $27(23.5 \%)$ & $7(6.1 \%)$ & $6(5.2 \%)$ & $41(35.7 \%)$ & $8(19.5 \%)$ \\
\hline Ketoprofen & $0(0.0 \%)$ & $1(0.9 \%)$ & $5(4.3 \%)$ & $0(0.0 \%)$ & $1(0.9 \%)$ & $7(6.1 \%)$ & $2(28.6 \%)$ \\
\hline Pyrazolone & $0(0.0 \%)$ & $0(0.0 \%)$ & $11(9.6 \%)$ & $2(1.7 \%)$ & $3(2.6 \%)$ & $16(13.9 \%)$ & $0(0.0 \%)$ \\
\hline Ibuprofen & $0(0.0 \%)$ & $0(0.0 \%)$ & $4(3.5 \%)$ & $0(0.0 \%)$ & $0(0.0 \%)$ & $4(3.5 \%)$ & $3(75.0 \%)$ \\
\hline $\mathrm{SUM}^{\star \star}$ & 0 & 5 & 134 & 13 & 34 & $115(100 \%)$ & $117(62.9 \%)$ \\
\hline
\end{tabular}

*erythema, maculopapular rashes, itching without a rash, and history of weakness or faintness

**Hypersensitivity reactions occurred after more than one drug in some individuals

Table 2 | Distribution of non-steroidal anti-inflammatory drug consumption after the oral provocation test and outcome. The data were obtained by a telephone survey.

\begin{tabular}{|c|c|c|c|c|}
\hline \multirow{2}{*}{ Substance } & \multicolumn{3}{|c|}{ Reaction to drug } & \multirow{2}{*}{ No. of subjects that took drug } \\
\hline & No reaction & Predictable adverse effect* & Hypersensitivity reaction & \\
\hline Paracetamol & $79(68.7 \%)$ & $0(0.0 \%)$ & $4(3.5 \%)^{\star \star}$ & $83(72.2 \%)$ \\
\hline Central analgesics & $11(9.6 \%)$ & $0(0.0 \%)$ & $2(1.7 \%)$ & $13(11.3 \%)$ \\
\hline Meloxicam & $2(1.7 \%)$ & $0(0.0 \%)$ & $0(0.0 \%)$ & $2(1.7 \%)$ \\
\hline Aspirin, $100 \mathrm{mg}$ only & $13(11.3 \%)$ & $1(0.9 \%)$ & $1(0.9 \%)$ & $15(13.0 \%)$ \\
\hline Aspirin, 500 mg & $23(20.0 \%)$ & $1(0.9 \%)$ & $0(0.0 \%)$ & $24(20.9 \%)$ \\
\hline Diclofenac & $17(14.8 \%)$ & $4(3.5 \%)$ & $3(2.6 \%)$ & $24(20.9 \%)$ \\
\hline Pyrazolone & $4(3.5 \%)$ & $0(0.0 \%)$ & $4(3.5 \%)$ & $8(7.0 \%)$ \\
\hline Other NSAIDs*** & $40(34.8 \%)$ & $1(0.9 \%)$ & $2(1.7 \%)$ & $43(41.0 \%)$ \\
\hline 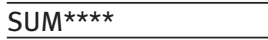 & & $8(7.0 \%)$ & $16(13.9 \%)$ & $115(100 \%)$ \\
\hline
\end{tabular}

${ }^{\star}$ The predictable adverse effects were nausea, vomiting, shivering, and nosebleed.

$\star \star$ None of the subjects had chronic urticaria.

***Thirty-three (28.7\%) subjects took naproxen, eight (7.0\%) subjects took ibuprofen, and two (1.7\%) subjects took ketoprofen. The predictable adverse effects were reported by a subject taking naproxen, and two subjects reported hypersensitivity reactions after taking naproxen and ibuprofen, respectively.

$\star \star \star \star$ Some subjects took more than one type of drug. 
aspirin $100 \mathrm{mg}$, one diclofenac). The NPV of the OPT for all analgesics was $96.4 \%$. The NPV of the OPT for aspirin was $97.4 \%$.

During the review of the hospital discharge letters, we found that $60(52.2 \%)$ subjects did not receive specific information concerning which analgesics were safe for them to use. Only $27.8 \%$ were told that aspirin was a safe drug for them to use.

Out of 115 subjects with a negative OPT for aspirin, 76 (66.1\%) did not retake aspirin over the next few years. The most common reasons were as follows: 30 (39.5\%) subjects did not need this particular drug, $28(36.8 \%)$ subjects feared a drug hypersensitivity reaction, and four (5.3\%) subjects were discouraged by their personal physician from re-administration due to an unclear instruction in the discharge letter. We did not obtain this information for $14(18.4 \%)$ subjects.

\section{Discussion}

A good concordance (86\%) was reported between the OPT with NSAIDs and the patients' history, at least for NERD (21). We were surprised to find a low number of positive OPTs in our cohort, particularly because we analyzed 115 selected subjects with a convincing medical history of hypersensitivity. It is possible that clinical history is good predictor of a positive OPT in patients with NERD and not in patients with NIUA or anaphylaxis, as were the vast majority of our patients. Indeed, several studies showed that the majority of patients with a convincing history of NSAID hypersensitivity were actually NSAID-tolerant. In a study by Zisa et al. of 159 patients with a clinical history of urticaria/angioedema apparently related to NSAIDs, only $10.7 \%$ were positive on the OPT with the suspected drugs (22). Half of these were single-NSAID reactors. Indeed, $37.1 \%$ of our patients were not challenged with the culprit drug but only with aspirin, which excluded intolerance to COX-1 inhibitors but not selective hypersensitivity to a single NSAID. As shown in a study performed by Chaudhry et al., $43 \%$ of homologous NSAID challenges but only $25 \%$ of heterologous NSAID challenges were positive in patients with a history of NSAID hypersensitivity (64\% had cutaneous reactions and 36\% had anaphylaxis) (23). Patients with anaphylaxis and those that reacted to diclofenac were most likely to have a positive challenge.

There are only a few reports in the literature on the NPV of OPTs with aspirin and NSAIDs. A French study published by Defrance et al. found that $53.3 \%$ of 260 patients followed up for a median time of 2.75 years re-took the tested drug; a hypersensitivity reaction was reported in $3.1 \%$, resulting in an NPV of the OPT of $98.6 \%$ (18). Our study covered an extended period of time (5.1 years) and found a similar NPT (96.4\%). We could speculate that many urticarial reactions following analgesic ingestion were not due to analgesic hypersensitivity but were provoked by the same under-

\section{References}

1. Mourad AA, Bahna SL. Hypersensitivities to non-steroidal anti-inflammatory drugs. Expert Rev Clin Immunol. 2014;10:1263-8.

2. Asero R. Oral aspirin challenges in patients with a history of intolerance to single non-steroidal anti-inflammatory drugs. Clin Exp Allergy. 2005;35:713-6.

3. Bommarito L, Zisa G, Riccobono F, Villa E, D’Antonio C, Calamari AM, et al. Avoidance of nonsteroidal anti-inflammatory drugs after negative provocation tests in urticaria/angioedema reactions: Real-world experience. Allergy Asthma Proc. 2014:35:303-6.

4. Kowalski ML, Makowska JS. Seven steps to the diagnosis of NSAIDs hypersensitivity: how to apply a new classification in real practice? Allergy Asthma Immunol Res 2015;7:312-20. lying cause that was the reason for taking the analgesic drug (i.e., viral infection). This speculation is supported by the fact that half of the reactions in our study occurred after paracetamol ingestion, which is commonly taken to treat symptoms of viral infections and is generally well tolerated in patients with COX-1 inhibitor hypersensitivity. A similar NPV was reported by Waton et al. (24). In this study, 65 patients with cutaneous symptoms during NSAID therapy were negative in the NSAID OPT. Eighteen (28\%) took the NSAID again and two reported hypersensitivity reactions, leading to a high NPV for the OPT.

In patients with a history of only cutaneous reactions and a negative OPT with NSAIDs, Bommarito et al. found that $47.7 \%$ did not re-take the tested NSAID (3). The main reason was fear of hypersensitivity reaction (70.8\%). In our study, the percentage of subjects that did not take the tested drug was even higher; one important reason for this was that the GPs discouraged the use of the NSAID due to the unclear discharge letter.

The mean provocative dose of oral aspirin that triggered respiratory reactions in people with asthma is $85.8 \mathrm{mg}(25)$. The threshold is even higher in patients with NSAID-induced urticaria/angioedema. The majority reacted only with a full therapeutic dose (22). One of the obstacles in the NSAID challenge test is desensitization, which might influence the OPT in such a way that the outcome can be a false negative $(19,20)$. Namely the protocol used for aspirin desensitization also uses a gradual increase in the dosage (doses are approximately doubled) at 90-minute intervals until a $500 \mathrm{mg}$ dose is reached.

Some subjects in our study re-took the culprit drug although it was not specifically tested with the OPT. This is particularly dangerous for diclofenac and pyrazolones, which are typical representatives of drugs that induce SNIUAA and should therefore be discouraged from re-administration unless a negative OPT with the culprit drug is reported $(17,26-29)$. In patients with hypersensitivity reactions to only one NSAID, alternative strong COX-1 inhibitors should be tested to determine whether the patients are single reactors and which NSAIDs are safe for them (30).

In conclusion, despite the negative OPT outcome, only $33.9 \%$ of subjects took aspirin. Only $0.9 \%$ experienced a hypersensitivity reaction with a drug that was negative in the OPT. In addition to performing the OPT, a clear explanation of the results is necessary in the discharge letter to ensure the safe use of analgesics by the patient.

\section{Acknowledgement}

We thank Ema Mušič, Mihaela Zidarn, Renato Eržen, Nissera Bajrović, Katja Adamič, and Nika Lalek for performing the diagnostic tests, and Julij Šelb for data management.
5. Makowska J, Lewandowska-Polak A, Kowalski ML. Hypersensitivity to aspirin and other NSAIDs: diagnostic approach in patients with chronic rhinosinusitis. Curr Allergy Asthma Rep. 2015;15:552.

6. Nabavi M, Esmaeilzadeh H, Arshi S, Bemanian MH, Fallahpour M, Bahrami A, et al. Aspirin hypersensitivity in patients with chronic rhinosinusitis and nasal polyposis: frequency and contributing factors. Am J Rhinol Allergy. 2014;28:239-43.

7. Abuaf N, Rostane H, Barbara J, Toly-Ndour C, Gaouar H, Mathelier-Fusade P, et al. Comparison of CD63 upregulation induced by NSAIDs on basophils and monocytes in patients with NSAID hypersensitivity. J Allergy (Cairo). 2012;2012:580873. 
8. Campo P, Ayuso P, Salas M, Plaza MC, Cornejo-García JA, Doña I, et al. Mediator release after nasal aspirin provocation supports different phenotypes in subjects with hypersensitivity reactions to NSAIDs. Allergy. 2013;68:1001-7.

9. Matsuo H, Yokooji T, Morita H, Ooi M, Urata K, Ishii K, et al. Aspirin augments IgEmediated histamine release from human peripheral basophils via Syk kinase activation. Allergol Int. 2013;62:503-11.

10. Neighbour H. Mechanisms of aspirin-intolerant asthma: identifying inflammatory pathways in the pathogenesis of asthma. Int Arch Allergy Immunol. 2014;163:1-2.

11. Kowalski ML, Asero R, Bavbek S, Blanca M, Blanca-Lopez N, Bochenek G, et al. Classification and practical approach to the diagnosis and management of hypersensitivity to nonsteroidal anti-inflammatory drugs. Allergy. 2013;68:1219-32.

12. Cormican LJ, Farooque S, Altmann DR, Lee TH. Improvements in an oral aspirin challenge protocol for the diagnosis of aspirin hypersensitivity. Clin Exp Allergy. 2005;35:717-22.

13. Malskat WS, Knulst AC, Bruijnzeel-Koomen CA, Röckmann H. Tolerance to alternative cyclooxygenase- 2 inhibitors in nonsteroidal anti-inflammatory drug hypersensitive patients. Clin Transl Allergy. 2013;3:20.

14. Doña I, Blanca-López N, Jagemann LR, Torres MJ, Rondón C, Campo P, et al. Response to a selective COX-2 inhibitor in patients with urticaria/angioedema induced by nonsteroidal anti-inflammatory drugs. Allergy. 2011;66:1428-33.

15. Rutkowski K, Nasser SM, Ewan PW. Paracetamol hypersensitivity: clinical features, mechanism and role of specific IgE. Int Arch Allergy Immunol. 2012;159:60-4.

16. Himly M, Jahn-Schmid B, Pittertschatscher K, Bohle B, Grubmayr K, Ferreira F, et al. IgE-mediated immediate-type hypersensitivity to the pyrazolone drug propyphenazone. J Allergy Clin Immunol. 2003;111:882-8.

17. Picaud J, Beaudouin E, Renaudin JM, Pirson F, Metz-Favre C, Dron-Gonzalvez M, et al. Anaphylaxis to diclofenac: nine cases reported to the Allergy Vigilance Network in France. Allergy. 2014;69:1420-3.

18. Defrance C, Bousquet PJ, Demoly P. Evaluating the negative predictive value of provocation tests with nonsteroidal anti-inflammatory drugs. Allergy. 2011;66:1410-4.

19. Comert S, Celebioglu E, Yucel T, Erdogan T, Karakaya G, Onerci M, et al. Aspirin 300 $\mathrm{mg} /$ day is effective for treating aspirin-exacerbated respiratory disease. Allergy. 2013;68:1443-51.
20. White AA, Bosso JV, Stevenson DD. The clinical dilemma of "silent desensitization" in aspirin-exacerbated respiratory disease. Allergy Asthma Proc. 2013;34:378-82.

21. Dursun AB, Woessner KA, Simon RA, Karasoy D, Stevenson DD. Predicting outcomes of oral aspirin challenges in patients with asthma, nasal polyps, and chronic sinusitis. Ann Allergy Asthma Immunol. 200;100:420-5.

22. Zisa G, Riccobono F, Bommarito L, D’Antonio C, Calamari AM, Poppa M, et al. Provocation tests with the offending nonsteroidal anti-inflammatory drugs in patients with urticaria/angioedema reactions. Allergy Asthma Proc. 2012;33:421-6.

23. Chaudhry T, Hissaria P, Wiese M, Heddle R, Kette F, Smith WB. Oral drug challenges in non-steroidal anti-inflammatory drug-induced urticaria, angioedema and anaphylaxis. Intern Med J. 2012;42:665-71.

24. Waton J, Pouget-Jasson C, Loos-Ayav C, Trechot P, Bursztejn AC, Schmutz JL, et al. Drug re-challenges in cutaneous adverse drug reactions: information and effectiveness in the long-term management of patients. Allergy. 2011;66:941-7.

25. Morales DR, Guthrie B, Lipworth BJ, Jackson C, Donnan PT, Santiago VH. NSAID-exacerbated respiratory disease: a meta-analysis evaluating prevalence, mean provocative dose of aspirin and increased asthma morbidity. Allergy. 2015;70:828-35

26. Harrer A, Lang R, Grims R, Braitsch M, Hawranek T, Aberer W, et al. Diclofenac hypersensitivity: antibody responses to the parent drug and relevant metabolites. PLoS One. 2010;5:e13707.

27. Kowalski ML, Woszczek G, Bienkiewicz B, Mis M. Association of pyrazolone drug hypersensitivity with HLA-DQ and DR antigens. Clin Exp Allergy. 1998;28:1153-8.

28. Kowalski ML, Bienkiewicz B, Woszczek G, Iwaszkiewicz J, Poniatowska M. Diagnosis of pyrazolone drug sensitivity: clinical history versus skin testing and in vitro testing. Allergy Asthma Pro. 1999;20:347-52.

29. De Pasquale T, Buonomo A, Illuminati I, D’Alò S, Pucci S. IgE-mediated anaphylaxis to ketoprofen: a case report. J Investig Allergol Clin Immunol. 2015;25:79-80.

30. Asero R. Use of ketoprofen oral challenges to detect cross-reactors among patients with a history of aspirin-induced urticaria. Ann Allergy Asthma Immunol. 2006;97:187-9. 